\title{
Cluster Head Election Approach based on Weighted Clustering Algorithm for MANET
}

\author{
Vaishali Chourey \\ Associate Professor \\ Department of Computer Science and Engineering \\ Medicaps Institution of Technology and \\ Management Indore, M.P.
}

\author{
Paridhi Kala \\ Research Scholar \\ Department of Computer Science and Engineering \\ Medicaps Institution of Technology and \\ Management Indore, M.P.
}

\begin{abstract}
The mobile ad hoc network is self-configurable network technology where not any fixed infrastructure is available. Due to frequent mobility and dynamic topology the network suffers from the frequent path breaks and the connectivity issues. The clustering techniques are utilized to improve the connectivity and scalability of network. Therefore a new weight based clustering algorithm is developed using the remain energy, signal to noise ratio, mobility and connectivity for better performance in a cluster. The implementation and the comparative performance study are performed in network simulator 2 platforms. The performance studies of the technique are given in terms of packet delivery ratio, remaining energy, end to end delay, throughput and overhead in network. The obtained results demonstrate the proposed technique improves the reliability of cluster.
\end{abstract}

\section{Keywords}

MANETs, Clustering, Cluster head, packet delivery ratio

\section{INTRODUCTION}

Mobile ad hoc network or MANET is a class of wireless network. In which the network devices utilize the wireless connectivity for communication. The mobile ad hoc network support the mobility and ad hoc network configuration. Mobile ad hoc network suffers from the performance as well as the security issues due to their adoptive and ad hoc nature. Due to the network's ad hoc nature, the new nodes can join the network and any node can leave the network at any time. The node's limited radio range affects the network scaling and the connectivity. Different clustering algorithms are implemented to support scaling of network and their performance. In order to provide efficient communication there need to be a wireless backbone. The backbone must change to reflect the changes in the network topology as the nodes move. The algorithm to choose the backbone should be very fast and efficient as they involve mobile nodes powered by battery. Clustering is also a solution to the above problem[1]. Even clustering suffers overhead from cluster formation and maintenance. Since the nodes are powered by limited supply of power, the objective is to enhance the existing clustering algorithms to make them a bit more efficient. Out of these the greatest challenge for an Ad Hoc network is resource management and that too particularly the battery life which, later in the thesis is referred to as battery power. The lifetime of an Ad Hoc network can be defined as the moment when the network starts working till one of the node drops out of the network because the battery was depleted. When clusters are formed in the network, one of the nodes becomes the cluster head (there could also be multiple cluster head depending on the selection parameters), which shoulders the responsibility of maintaining communication with all the other nodes. This responsibility of maintaining the communication links depletes the battery very fast as this node has to make more number of communications as compared to a normal node and the node drops out of the network. This reduces the network lifetime. In this thesis we discuss different clustering algorithms and propose an enhancement for cluster head selection algorithm.

The key aim of the proposed work is to investigate the different approaches of the network and the network performance scaling. Cluster head selection techniques are required to maintain the cluster and its performance. In addition of that using the traditional efficient techniques a new method is also required to develop which provide efficient routing support and network scaling efficiently. The clustering is a technique by which the entire network nodes are arranged in groups where the most responsible node is cluster head. This cluster head provide the connectivity support and other monitoring supports. The proposed work is focused on finding the most optimum technique for network clustering and cluster head selection. The cluster head selection algorithm of the traditional approach is improved using some different node quality parameters. The Weighted Clustering Algorithm is implemented with the quality parameters like Energy, Connectivity, Mobility and signal to noise ratio. These node parameter improves the performance of the network from the traditional approach. The performance of the network is analyzed on the basis of end to end delay, packet delivery ratio, remaining energy, throughput and overhead.

\section{RELATED WORK}

Mobile Ad-Hoc network (MANET) is a type of communication network which is used for data communication between mobile nodes using wireless channels. Clustering has evolved as an important research topic in MANETs as it improves the system performance of large MANETs. Clustering is a process that divides the network into nodes groups, called clusters. Each cluster has a Cluster Head $(\mathrm{CH})$ as coordinator within the sub-structure. A clustering technique for MANETs, which is weighted clustering algorithm is given in [1]. The performances of this algorithm are evaluated through simulation and the results are encouraging. A clustering technique is used to adapt the solution of energy consumption. Reviews of most clustering techniques that improves the power conservative in mobile ad hoc network and increase the battery usage in ad hoc wireless network devices and conserve the power energy consumption [3]. A distributed weighted clustering algorithm for MANETs [4], this approach is based on combined weight metric that takes into account several system parameters like the node degree, transmission range, energy and mobility of the nodes. Enhanced ILP formulations for the Clustering Problem, through the enablement of multi-hop connections and intracluster communication, and assesses the performance of stateof-the art generic ILP and SAT solvers in solving the 
enhanced formulations [5] . The reactive power-alert technique for communication between ad hoc network nodes by continuously alerting their energy status to neighbor nodes [6].Here the concentration is on reducing the energy consumption by proposing optimal path selection method. An extensive literature survey of MANET clustering algorithm was done and lastly they proposed an algorithm for cluster head selection and cluster maintenance [7]. They proposed algorithm which uses self-organizing principle for binding a node with a cluster, and can reduce the explicit message passing in cluster maintenance. Thus, there is no need of extra message passing during further cluster maintenance. Unlike most usual methods, a cluster head in their proposed algorithm acts only as an identifying tag for cluster entity, further it performs as a typical node when it is acting as head. In mobile ad-hoc networks (MANET), the movement of the nodes may quickly change the networks topology resulting in the increase of the overhead message in topology maintenance. The nodes communicate with each other by exchanging the hello packet and constructing the neighbor list at each node. MANET is vulnerable to attacks such as black hole attack, Gray hole attack, wormhole attack and Sybil attack. A black hole attack makes a serious impact on routing, packet delivery ratio, throughput, and end to end delay of packets. The performance comparison of clustering based and threshold based algorithms for detection and prevention of cooperative in MANETs is examined [21]. In this study every node is monitored by its own cluster head $(\mathrm{CH})$, while server (SV) monitors the entire network by channel overhearing method. Server computes the trust value based on sent and receives count of packets of the receiver node. It is implemented using AODV routing protocol in theNS2 simulations. The results are obtained by comparing the performance of clustering based and thresholds based methods by varying the concentration of black hole nodes and are analyzed in terms of throughput, packet delivery ratio. The results demonstrate that the threshold based method outperforms the clustering based method in terms of throughput, packet delivery ratio and end to end delay.

\section{PROPOSED WORK}

Mobile ad hoc network is self-organizing network where routing protocols are responsible for organizing the network infrastructure. In this network not a predefined infrastructure is available for communication and connectivity. The network devices use the wireless medium for communication. In this context wireless nodes are connected with each other using wireless links. The radio range of this communication links are limited therefore the frequent connection break and path loss is occurred. Additionally due to limitations of resources such as battery power and computational resources, this network is not much stable and scalable with increasing traffic loads. Therefore for optimizing the stable network performance clustering techniques provides ease in performance enhancement.

With the similar concept a promising technique is obtained in [1]. This technique is provide more stable clusters for the high mobility scenarios. But due to the backup cluster head and the monitoring of the backup cluster head the network frequently inject the additional packets thus the network performance in terms of routing overhead and the energy consumption is degraded. Therefore in this proposed work a new cluster head election algorithm is introduced for improving the performance of the traditional approach of cluster head selection. The proposed cluster head section algorithm promises to increase the packet delivery ratio, throughput and reduces the routing overhead, end to end delay ,energy consumption of the traditional concept of cluster head selection.

\section{CLUSTER HANDLING}

The proposed cluster head selection technique is depend on the node quality estimation. Using the node's quality a weight is estimated and the higher obtained weighted node is selected as the cluster head. In addition of that for providing the stability in the clusters the cluster head selection is fixed in a defined time interval. Therefore the entire methodology of the cluster head selection technique is divided in three major modules as described.

4.1 Node parameter selection As stated before the proposed cluster head selection process is depends on the node's quality and their performance. Therefore the following node properties are incorporated for efficient node selection.

1. Energy: The ad hoc network devices are developed with the inbuilt energy source says the battery. The battery provides support to the node's for a limited amount of time. If the nodes having the low energy than the network nodes are not functioning properly, therefore energy efficient node's selection is required for stable clustering.

2. Connectivity: If the node is connected with a significant amount of the neighbor nodes than that node can serve for all the neighbor nodes. Therefore that is an essential parameter for node's selection.

3. Mobility: The ad hoc network supports the frequent mobility therefore frequent path break and connection losses are occurred. For stable clustering the less mobility nodes are required which provide the continuous connectivity among the nodes.

4. SNR: Signal to noise ratio in communication represents the amount of error in the propagated signals among the two communicating nodes. if this ratio is less than the nodes are efficiently communicate each other and retransmission of data is reserved. Thus low SNR nodes are selected for efficient cluster formation.

This section demonstrates the different performance parameters of node by which the nodes are evaluated for cluster head selection. The next section introduces the technique for network clustering.

\subsection{Network clustering}

The proposed weight based clustering technique is depend on the node quality and the efficient node is defined as the cluster head. Therefore good node having the higher weights thus for node evaluation for clustering is given as:

1. Compute the remain energy of nodes using the given formula and during further solution design that can be denoted using symbol E remain energy = initial energy consumed energy

2. Connectivity is computed using the number of neighbors available for the targeted node and for further discussion that is denoted using the symbol $\mathrm{C}$

3. Obtain the comparative mobility of node that can be computed using the following formula

$$
M=\frac{1}{T} \sum_{i=1}^{T} \sqrt{\left(X_{t}-X_{t-1}\right)^{2}+\left(Y_{t}-Y_{t-1}\right)^{2}}
$$


4. SNR of a target node can be computed using the following formula and for further discussion that is denoted by $\mathrm{S}$

$$
S N R=10 \log _{10}\left(\frac{R^{2}}{M S E}\right)
$$

Finally for the weight calculations the estimated parameters are combined with each other using the following formula

$$
W=w_{1} * E+w_{2} * C+w_{3} * M+w_{4} * S
$$

The weight $\mathrm{W}$ is a common property of the given node and the factors $w_{1} \ldots w_{4}$ are the weight factors that are having a value among $0-1$. These factors are scale the obtained values of network parameters and that is also required to have the sum of these values are exactly 1 . In further discussion the node parameters are used for cluster head selection.

\subsection{Cluster head selection}

Now the entire network nodes are evaluated and their obtained weights are compared to each other, when the most optimum nodes are discovered than that is labeled as the cluster head. That process can be summarized using the following steps:
1. For every time interval $\mathrm{T}$ repeat
2. Each node finds its neighbors and builds its neighbor-hood table.
3. Each node calculates its weight by calling the weight calculation algorithm given above.
4. Each node broadcasts its weight to its neighbors. If it has maximum weight among its neighbors are selected as the cluster head.
5. The node with maximum weight broadcasts cl- head message to other nodes.
6. End for

\section{SIMULATION AND RESULT ANALYSIS}

\subsection{Simulation Setup}

This section provides the desired network configuration for simulation of the proposed scheme for cluster head selection process implementation using AODV routing protocol.

\begin{tabular}{|l|l|}
\hline Simulation properties & Values \\
\hline Antenna model & Omni Antenna \\
\hline Dimension & 750 X 550 \\
\hline Radio-propagation & Two Ray Ground \\
\hline Channel Type & Wireless Channel \\
\hline No of Mobile Nodes & $10,20,30,40,50$ \\
\hline Routing protocol & \\
\hline
\end{tabular}

\begin{tabular}{|l|l|}
\hline Time of simulation & $10.0 \mathrm{Sec}$. \\
\hline
\end{tabular}

Table1. Network configuration set up

\subsection{Result analysis}

In order to demonstrate the effectiveness of the proposed algorithm over the traditional approach of the cluster head selection there are two different network scenarios are provided.

\subsubsection{Simulation Scenario}

1. Simulation using the traditional approach: in this phase the maximized reliability clustering algorithm is implemented in a mobile ad hoc network environment and their performance by increasing the number of nodes are evaluated.

2. Simulation using the proposed algorithm: in this simulation scenario the proposed algorithm is implemented through modifications on the AODV routing protocol and the performance of the network is evaluated with increasing the number of nodes in network. The obtained performance is further compared with the traditional approach previously implemented to the system.

After implementation of both the experimental scenarios the following facts are observed for network performance and functioning.

1. The traditional cluster head selection algorithm creates two different cluster heads first is primary and second the backup cluster. When the primary cluster head is crashed then the secondary cluster head is labeled as cluster head. In addition of that this newly elected cluster head recommend the new cluster head.

2. During the proposed method simulation using the node quality the cluster head is elected and in the regular time interval the cluster head election process is taken place again these process needs less time and less routing overhead.

\subsubsection{End to end delay}

End to end day on network refers to the time taken for a packet to be transmitted across a network from source to destination device.

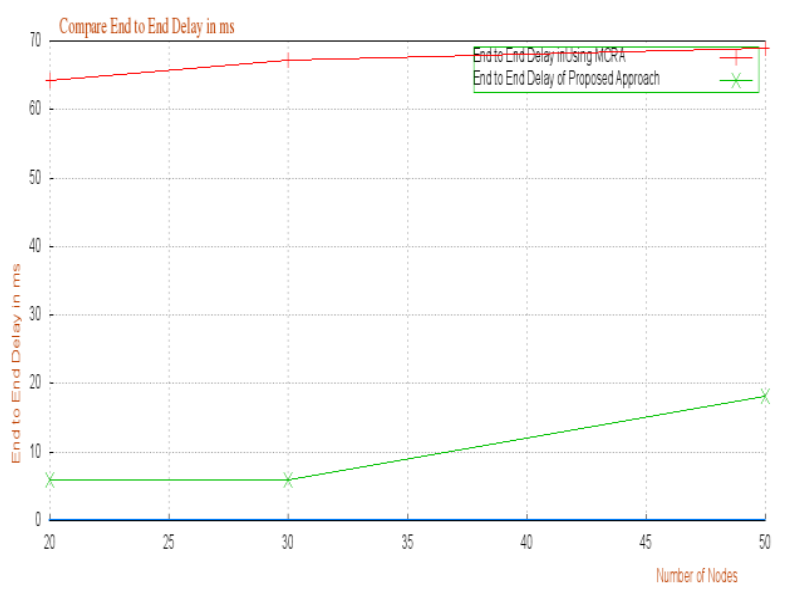

Figure 1. end to end delay 
The comparative performance of the previously available algorithm and the proposed algorithm is given using figure 1 in this diagram the $\mathrm{X}$ axis shows the number of nodes in network and the $\mathrm{Y}$ axis shows the end to end delay in network in terms of milliseconds. According to the obtained results from both the algorithms the proposed algorithm needs less time for delivering the data as compared to the traditional method.

\subsubsection{Remain energy}

The ad hoc devices has the limited energy because the device has inbuilt energy source (battery). During each event in network the node consumes a fixed amount of energy from their initial energy. The remaining energy of the nodes in different experiments are given using figure 2 . in this experiment the number of nodes are demonstrated in $\mathrm{X}$ axis and the relative performance (remaining energy) is given in $\mathrm{Y}$ axis.

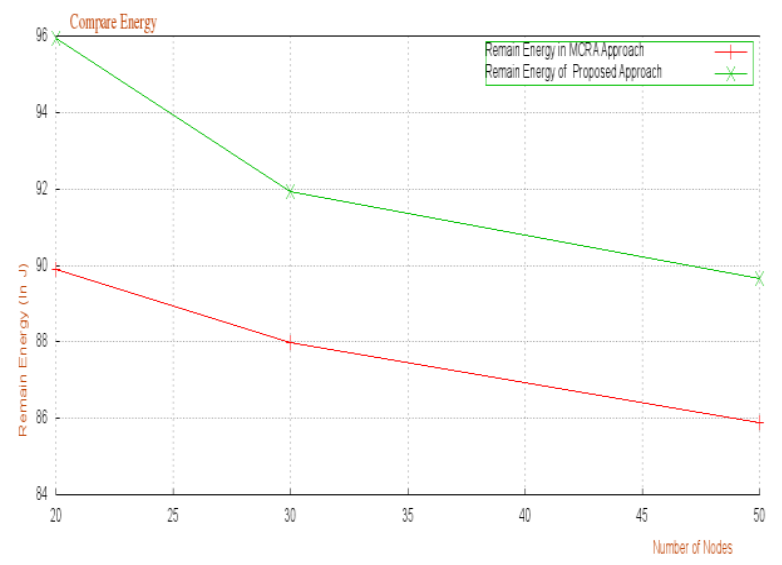

Figure 2. remain energy

The proposed algorithm (green line) preserves more energy as compared to the traditional approach (red line). Therefore the proposed approach is much efficient than the traditional approach of the cache management.

\subsubsection{Packet delivery ratio}

Packet delivery ratio provides information about the performance of any routing protocols, where PDR is estimated using the formula given

$$
\text { packetdeliveryratio }=\frac{\text { total delivered packets }}{\text { total sent packets }}
$$

The comparative packet delivery ratio of the network is given using figure 3 in this diagram the proposed algorithm's performance is represented using the green line and the red line shows the performance of the traditional cache management technique. for representing the performance of network the $\mathrm{Y}$ axis contains the percentage packets delivered and the $\mathrm{X}$ axis contains the number of nodes in network experimentations. According to the demonstrated results the proposed approach delivers more packets than the traditional approach of the cluster head management.

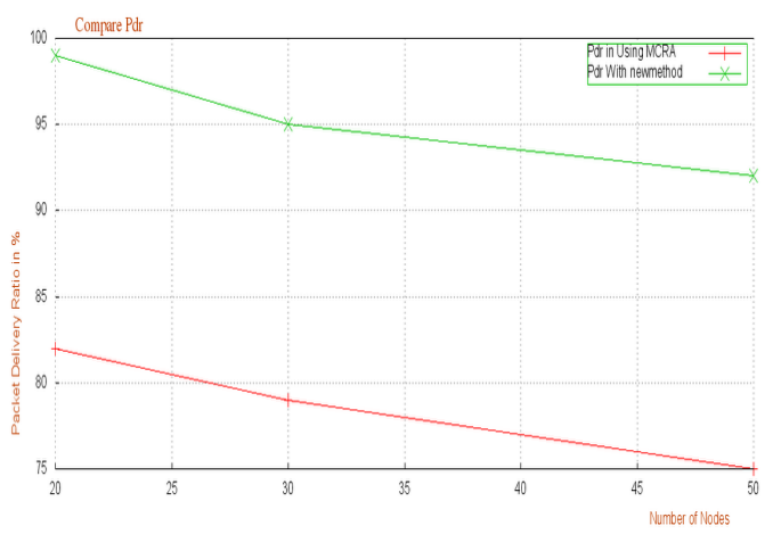

Figure 3. packet delivery ratio

\subsubsection{Throughput Comparison}

The comparison of throughput of the network is given using figure 4 in this diagram the proposed algorithm's performance is represented using the green line and the red line shows the performance of the traditional cache management technique. for representing the performance of network the $\mathrm{Y}$ axis contains the throughput and the $\mathrm{X}$ axis contains the number of nodes in network experimentations. According to the demonstrated results the proposed approach throughput is more than the traditional approach of the cluster head management.

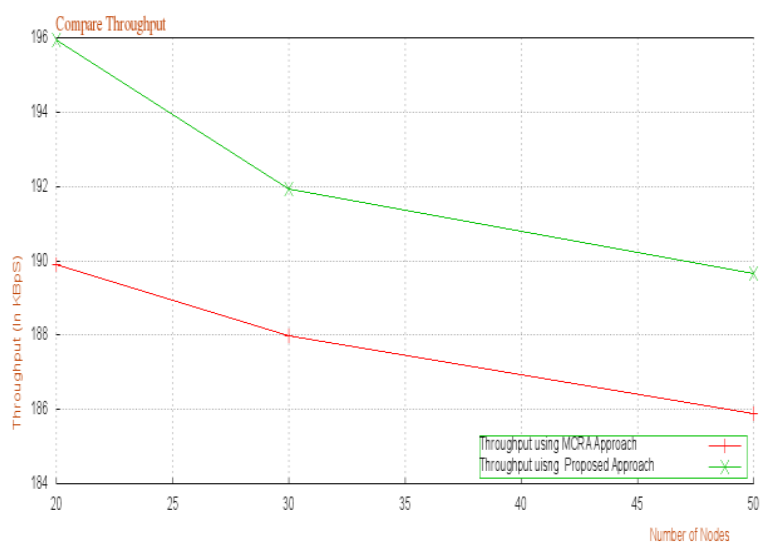

Figure 4. throughput comparison

\subsubsection{Over Head Comparison}

The overhead comparison of the network is given using figure 5.5 in this diagram the proposed algorithm's performance is represented using the green line and the red line shows the performance of the traditional clustering technique. For representing the performance of network the $\mathrm{Y}$ axis contains the overhead and the $\mathrm{X}$ axis contains the number of nodes in network experimentations. According to the demonstrated results the proposed approach provides less overhead than the traditional approach of the cluster head management. 


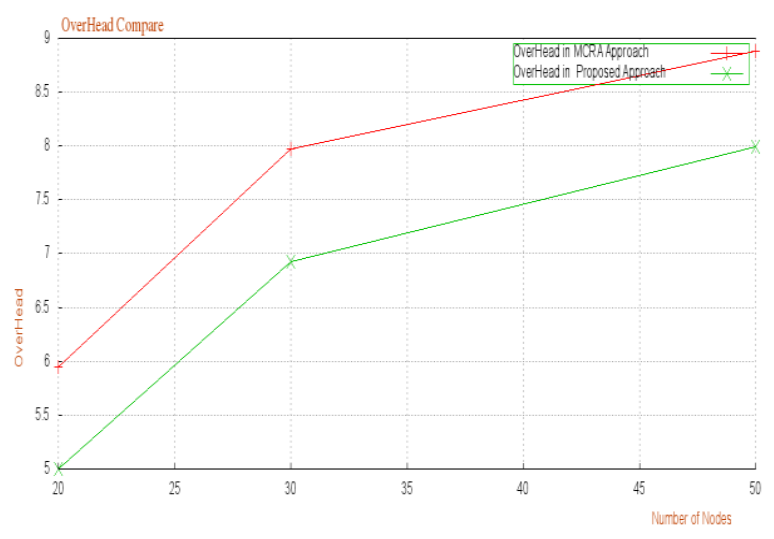

Figure 5 overhead comparison

The performance of the proposed and traditional cluster head management technique is evaluated in terms of packet delivery ratio, end to end delay and the remaining energy, overhead, and throughput. The performance summary of the proposed cluster head election algorithm is given using table 2.The table shows the values of the parameter compared. This will help us to easily analyze the facts of the traditional technique and proposed technique.

\begin{tabular}{|l|l|l|l|}
\hline $\begin{array}{l}\text { S. } \\
\text { no. }\end{array}$ & Parameters & $\begin{array}{l}\text { Traditional } \\
\text { technique }\end{array}$ & $\begin{array}{l}\text { Proposed } \\
\text { technique }\end{array}$ \\
\hline 1 & $\begin{array}{l}\text { Packet delivery } \\
\text { ratio }\end{array}$ & $71.9 \%$ & $81.2 \%$ \\
\hline 2 & End to end delay & $15 \mathrm{~ms}$ & $9 \mathrm{~ms}$ \\
\hline 3 & Remain Energy & $78.8(\mathrm{~J})$ & $82.2(\mathrm{~J})$ \\
\hline 4 & Throughput & $145 \mathrm{kbps}$ & $159 \mathrm{kbps}$ \\
\hline 5 & Overhead & 8.2 & 6.9 \\
\hline
\end{tabular}

Table 2. performance summary

According to the given description in table 2 the performance of the proposed technique is much adoptable as compared to the traditional approach of cluster head selection process.

\section{CONCLUSIONS}

The mobile ad hoc network is one of the most frequently used technologies for the rapid configurable network. Due to their ad hoc nature of topology development and mobility this network technology is very popular in army based applications and disaster management. But due to frequently changing topology and mobility the network suffers from the performance and connectivity issues. In this proposed work the performance and network scaling technologies are discussed and a promising technique is obtained as given in [1]. This protocol implemented with the backup cluster head algorithm during the loss of the primary cluster head this backup cluster head provides the support for connectivity.
But due to frequent control message exchange the network suffers from the high routing overhead. For this a new concept of the cluster head election technique is provided in this study. The proposed cluster head election algorithm first evaluates the nodes quality in terms of energy, connected nodes, mobility and SNR. During the fixed amount of time interval the process of cluster head election is repeated in continuation. This process increases the packet delivery ratio in the cluster head and is retained more for longer span of time. The throughput of the network is increased. Finally, the network files. The performance of the proposed and traditional cluster head management technique is evaluated in terms of packet delivery ratio, end to end delay overhead is reduced.

The proposed technique's implementation is performed using the network simulator 2 environment.After simulation the performance of the network is evaluated using network generated trace, throughput overhead and remaining energy. The performance summary of the proposed cluster head election algorithm is given using table 2 .

According to the given description the performance of the proposed technique is much adoptable as compared to the traditional approach of cluster head selection process.

The proposed technique is implemented successfully and the performance of the given technique is also compared with the relevant algorithm. The result shows the effective performance of the given algorithm. In near future the more literature is collected to enhance the given methodology more in terms of throughput.

\section{REFERENCES}

[1] Feng Jiang, LanlanRui, Yaoyong Guo, Xuesong Qiu, Wei Li, "Reliability-Oriented Clustering Algorithm for Service Search in Ubiquitous Stub Environments", Copyright IEICE - Asia-Pacific Network Operation and Management Symposium (APNOMS) 2014

[2] Wojciech Bednarczyk, Piotr Gajewski, "An Enhanced Algorithm for MANET Clustering Based on Weighted Parameters", Universal Journal of Communications and Network 1(3): 88-94, 2013

[3] Dr. Mohammad U. Bokhari, Hatem S. A. Hamatta, Shams Tabrez Siddigui, "A Review of Clustering Algorithms as Applied in MANETs", International Journal of Advanced Research in Computer Science and Software Engineering, Volume 2, Issue 11, November 2012

[4] Naveen Chauhan, Lalit Kumar Awasthi, Narottam Chand, Vivek Katiyar, Ankit Chugh, "A Distributed Weighted Cluster Based Routing Protocol for MANETs", Wireless Sensor Network, 2011, 3, 54-60

[5] Syed Zahidi, Fadi Aloul, Assim Sagahyroon, Wassim ElHajj, "Using SAT \& ILP Techniques To Solve Enhanced ILP Formulations Of The Clustering Problem In MANETS", 978-1-4577-1379-8/12/\$26.00 @2012 IEEE

[6] Shilpa Bade, Meeta Kumar, Pooja Kamat, "A Reactive Energy-Alert Algorithm for MANET and Its Impact on Node Energy Consumption", International Journal of Computer Applications (0975 - 8887) Volume 71No.18, June 2013

[7] Soumyabrata Talapatra, Alak Roy, "Mobility Based Cluster Head Selection Algorithm for Mobile Ad-Hoc Network", I.J. Computer Network and Information 
Security, 2014, 7, 42-49 Published Online June 2014 in MECS

[8] Prerna Malhotra, Ajay Dureja, "A Survey of WeightBased Clustering Algorithms in MANET", IOSR Journal of Computer Engineering (IOSR-JCE) e-ISSN: 22780661, p- ISSN: 2278-8727Volume 9, Issue 6 (Mar. - Apr. 2013), PP 34-40

[9] Hui Cheng, Shengxiang Yang, Jiannong Cao, "Dynamic genetic algorithms for the dynamic load balanced clustering problem in mobile ad hoc networks", Expert Systems with Applications 40 (2013) 1381-1392, 2012 Elsevier Ltd. All rights reserved.

[10] S. A. Ade \& P. A. Tijare, "Performance Comparison of AODV, DSDV, OLSR and DSR Routing Protocols in Mobile Ad Hoc Networks", International Journal of Information Technology and Knowledge Management, July-Dec 2010, Volume 2, No. 2, pp. 545-548

[11] Vinay Sridhara, Nagendra Subramanya, "Evaluating Different Techniques to Improve TCP Performance over Wireless Ad Hoc Networks", http://citeseerx.ist.psu.edu/viewdoc/summary?doi=10.1.1 .80 .8842

[12] M. S. karthikeyan, K. Angayarkanni, and Dr. S. Sujatha, "Throughput Enhancement in Scalable MANETs using Proactive and Reactive Routing Protocols", proceedings of the international multi conference of engineering and computer scientists, Vol II, march 2010

[13] J. Broch, D. A. Maltz, D. B. Johnson, Y.C. Hu and J. Jetcheva, "A Performance Comparison of Multi-hop Wireless Ad-hoc Network Routing Protocols", Proceedings of the 4th Annual ACM/IEEE International Conference on Mobile Computing and Networking, PP. 85-97, ACM Press, 1998

[14] A. Boukerche, "Performance Comparison and Analysis of Ad-hoc Network Routing Algorithms", IEEE
Conference on Performance, Computing and Communication, PP.171-178, April 2001.

[15] Aniket Mathuriya, Pushpraj Pal and Love deep Grower, "Stability Aware Routing in Mobile Ad-Hoc Networks using Multiple Route", International Journal of Computers \& Technology, ISSN: 2277-3061, On line Volume 2 No. 3, June, 2012

[16] G. Ivascu, S. Pierre, A. Quintero, "QoS Support Based on a Mobile Routing Backbone for Ad-hoc Wireless Networks", Proceedings of the International journal Conference on Wireless Communications and Mobile Computing, IWCMC'06, PP. 121-126, Vancouver, Canada, July 2006

[17] A. Iwata, C. Chiang, G. Pei, M. Gerla, and T. Chen, "Routing Strategies for Ad-hoc Wireless Networks", IEEE Conference on Selected Areas in Communications, Vol. 17, No. 8, PP. 1369-1379, Japan, August 1999.

[18] P. Johnson, T. Larson, N. Headman, B. Mielczarek and M. Degmark, "Scenario Based Performance Analysis of Routing Protocols for Mobile Ad-hoc Networks", Mobicom'99, PP. 195-206, 1999.

[19] D. Chakeres and E. B. Royer "The Utility of Hello Message for Determining Link Connectivity", Proceedings of 5th International Conference on Wireless Personal Multimedia Communications (WPMC) Vol. 2 , PP. 504-508, Hawaii, 2002.

[20] P. S. Hiremath, Anuradha T., "Performance Comparison of Cluster based and Threshold based Algorithms for Detection and Prevention of Cooperative Black Hole Attack in MANETs", Int. J. Advanced Networking and Applications Volume: 6 Issue: 3 Pages: 2352-2358 (2014) ISSN : 0975-0290 